\section{P 030 THE COMMUNITY CARE PATHWAYS AT THE END OF LIFE (CAPE) STUDY- EARLY FINDINGS}

Sue Boase, Emily Moran, Stephen Barclay. The Primary Care Unit, Institute of Public Health, University of Cambridge, United Kingdom

10.1136/bmjspcare-2014-000654.71

Background Current NHS End of Life Care policy favours raising the proportion of deaths that occur at home. Currently this only occurs in $21.8 \%$ of the total. Although most people would like to die at home, research has shown that people's preferences are influenced by the progression of their illness, how much support is available and their social circumstances.

Aims The aim of the study is to clarify for whom home death is "good" and for whom it is not, and to ascertain whether the focus on place of death is helpful.

Methods Using a mixed-method approach, data on 400 deceased patients, the perceptions of their GPs, community nurses and bereaved carers are brought together to generate an understanding of patterns of care need, and pathways of care provision at the end of life.

Results Initial findings indicate the importance of trust and communication among the team. The health professionals felt that noncancer patients are more difficult to care for than those with cancer, who have a clearer trajectory. Not all admissions to hospital at the end of life were seen as negative, and it may be the preferred place of care and death for some patients. Different agencies being on different electronic systems can cause communication difficulties and the different End of Life Care initiatives can be time-consuming and confusing.

Conclusions Although the findings are preliminary at present, it is hoped that this study will shed light on the investment of time and resources and the logistics of providing care at the end of life in the community whether the place of death is at home, in a care home, hospice or hospital. In turn this aims to address the question of whether the focus on place is the best measure of a "good" death. 